\title{
A model displaying extremely inhomogeneous matter distribution in General Relativity
}

\author{
Elcio Abdalla and Cecilia B. M. H. Chirenti \\ Instituto de Física, Universidade de São Paulo \\ C.P.66.318, CEP 05315-970, São Paulo, Brazil.
}

\begin{abstract}
We consider a toy metric in four dimensional space-time defined in terms of a recursive hierarchical prescription. The matter distribution turns out to be extremely inhomogeneous. Surprisingly, for very large samples the average mass density tends (very slowly) to a constant. There is no trace of fractal dimension left.
\end{abstract}

Although most authors tend to believe that the large scale distribution of matter should be constant as predicted by Einstein's cosmological principle [1] there is also a belief that such an idea should be further checked against observation [2. Some authors argued quite convincingly that the large scale distribution of matter should be fractal, quite the oposite to that expected by the cosmological principle. Inhomogeneous cosmological models have been searched by many authors [3] [4 [5] [6] [7] 8]. The fact that detailed maps show quite strong inhomogeneities has been a motivation for long controversies 9, 10.

Here, we construct a metric defined in terms of a hierarchy, building an expectation that the matter distribution obtained from the corresponding Einstein's Equations are extremely inhomogeneous, contradicting the cosmological principle, and probably presenting fractality properties, with a non trivial effective dimension. As it turns out, some of these conclusions are false, as we shall see.

Our starting point is the diagonal metric defined in terms of a distribution of points. A point in space-time is only defined at the integers. Indeed, we expect that a metric describing a fractal distribution cannot be continuous at any point, therefore it can only be defined on a discrete set. With this motivation in mind, we define, in a given direction $i$ (either 1,2 or 3 ) the 
distance between two points as pictorically represented in Fig. 1, with the metric

$$
g_{\mu \nu}=\left(\begin{array}{cccc}
1 & 0 & 0 & 0 \\
0 & g_{11} & 0 & 0 \\
0 & 0 & g_{22} & 0 \\
0 & 0 & 0 & g_{33}
\end{array}\right),
$$

where the metric is defined on all integers, depending on their decomposition in terms of powers of 2 , as below,

$$
\begin{aligned}
& g_{11}(x)=a^{2 k}, \quad \text { with } \quad x=2^{k+1}+2^{k}-1 \\
& g_{22}(y)=a^{2 l}, \quad \text { with } y=2^{l+1}+2^{l}-1 \\
& g_{33}(x)=a^{2 m}, \quad \text { with } z=2^{m+1}+2^{m}-1 .
\end{aligned}
$$

Above, $k, l$ and $m$ are nonnegative integers and the metric is defined as unit when the entry is an even number. This defines the metric (see Fig. 1).

Computing the Christoffel symbols and subsequently the curvature tensor requires some care, since we are not dealing with derivatives of functions, but differences of functions defined on a discrete space. The result for the Christoffel symbols is

$$
\begin{aligned}
& \Gamma_{11}^{0}=-\frac{k}{a} g_{11} \frac{d a}{d t}, \quad \Gamma_{01}^{1}=\Gamma_{10}^{1}=\frac{k}{a} \frac{d a}{d t}, \quad \Gamma_{11}^{1}=\frac{(-1)^{x}}{2}\left(1-g^{11}\right), \\
& \Gamma_{22}^{0}=-\frac{l}{a} g_{22} \frac{d a}{d t}, \quad \Gamma_{02}^{2}=\Gamma_{20}^{2}=\frac{l}{a} \frac{d a}{d t}, \quad \Gamma_{22}^{2}=\frac{(-1)^{y}}{2}\left(1-g^{22}\right), \\
& \Gamma_{33}^{0}=-\frac{m}{a} g_{33} \frac{d a}{d t}, \quad \Gamma_{30}^{3}=\Gamma_{03}^{3}=\frac{m}{a} \frac{d a}{d t}, \quad \Gamma_{33}^{3}=\frac{(-1)^{z}}{2}\left(1-g^{33}\right) \text {. }
\end{aligned}
$$

A naive construction of the Riemann tensor may lead to a result incompatible with the general symmetry properties required for that tensor [11]. We thus define it as

$$
R_{\lambda \mu \nu \kappa}=\frac{1}{2}\left[\frac{\partial^{2} g_{\lambda \nu}}{\partial x^{\kappa} \partial x^{\mu}}-\frac{\partial^{2} g_{\mu \nu}}{\partial x^{\kappa} \partial x^{\lambda}}-\frac{\partial^{2} g_{\kappa \lambda}}{\partial x^{\mu} \partial x^{\nu}}+\frac{\partial^{2} g_{\mu \kappa}}{\partial x^{\lambda} \partial x^{\nu}}\right]+g_{\eta \sigma}\left[\Gamma_{\lambda \nu}^{\eta} \Gamma_{\mu \kappa}^{\sigma}-\Gamma_{\lambda \kappa}^{\eta} \Gamma_{\mu \nu}^{\sigma}\right]
$$

which in the continuous version corresponds exactly to the usual definition, and here has the correct symmetry properties with respect to the interchange of indices. The Ricci tensor is readily obtained, with the result (the non diagonal components vanish)

$$
R_{00}=\frac{1}{a}(k+l+m) \frac{d^{2} a}{d t^{2}}+\frac{1}{a^{2}}\left(\frac{d a}{d t}\right)^{2}[k(k-1)+l(l-1)+m(m-1)],
$$




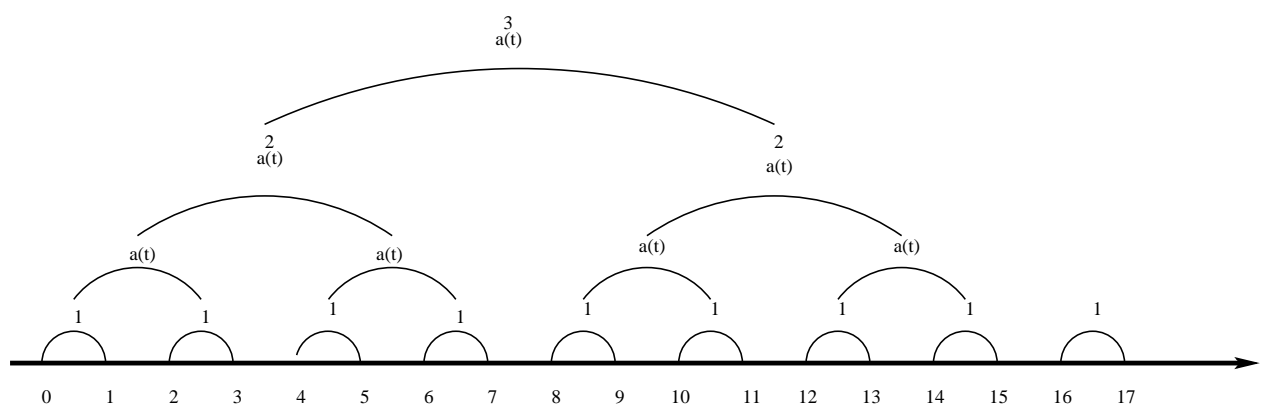

Figure 1: Diagram showing the definition of a hierarchical metric as a function of the distance. In Eq. 2 we defined a metric which realizes the above properties for nearby points. The arc segment with a power of $a$ shown here represents the distance between any element from one side of the arc and any element from the other side.

$$
\begin{aligned}
& R_{11}=g_{11}\left\{\frac{k}{a}+\frac{1}{a^{2}}\left(\frac{d a}{d t}\right)^{2}[k(k-1)+k l+k m]\right\} \\
& R_{22}=g_{22}\left\{\frac{l}{a}+\frac{1}{a^{2}}\left(\frac{d a}{d t}\right)^{2}[k l+l(l-1)+l m]\right\}
\end{aligned}
$$




$$
R_{33}=g_{33}\left\{\frac{m}{a}+\frac{1}{a^{2}}\left(\frac{d a}{d t}\right)^{2}[m k+m l+m(m-1)]\right\} .
$$

The matter density can be now readily calculated by means of the Einstein Equations, and we obtain

$$
T_{00} \equiv \rho=\frac{1}{8 \pi G}\left(\frac{1}{a} \frac{d a}{d t}\right)^{2}(k l+l m+m k) \equiv \rho_{0}(k l+l m+m k)
$$

The result shows severe inhomogeneities. A graph showing $\rho(x, y, 0)$ exemplifies the situation in Fig. 2.

We further questioned about the fact of whether such an inhomogeneous distribution could be described by a fractal. We numerically computed the limit

$$
\lim _{r \rightarrow \infty} \frac{N(r)}{r^{d}}
$$

where $N(r)=\sum_{0 \leq x, y, z \leq r} \rho(x, y, z)$ finding the value of $d$ where the limit exists. This is the usual definition of a fractal dimension [12]. Some results are sumarized in Figure 3.

It turned out, with some surprise, that the effective dimension of the distribution is $d=3$, that is, the hierarchical metric was not enough to lead to a hierarchical distribution of matter. Nonetheless, the matter distribution is very inhomogeneous, and does not seem to homogenize very soon. Whether such a simplified model can have any relation to the real matter distribution of the universe is not questioned, but the stability of the dimensionality of the effective distribution of matter is quite remarkable. This may give support to models displaying highly inhomogeneous behaviour either in the nonrelativistic domain, such as [6], or up to a certain cosmological distance, but smooth out their behaviour at large enough scales, as predicted by the cosmological principle, very slowly approaching the canonical value $d=3$ for the effective dimension.

Acknowledgements: This work has been supported by Fundação de Amparo à Pesquisa do Estado de São Paulo (FAPESP) and Conselho Nacional de Desenvolvimento Científico e Tecnológico (CNPq), Brazil. 


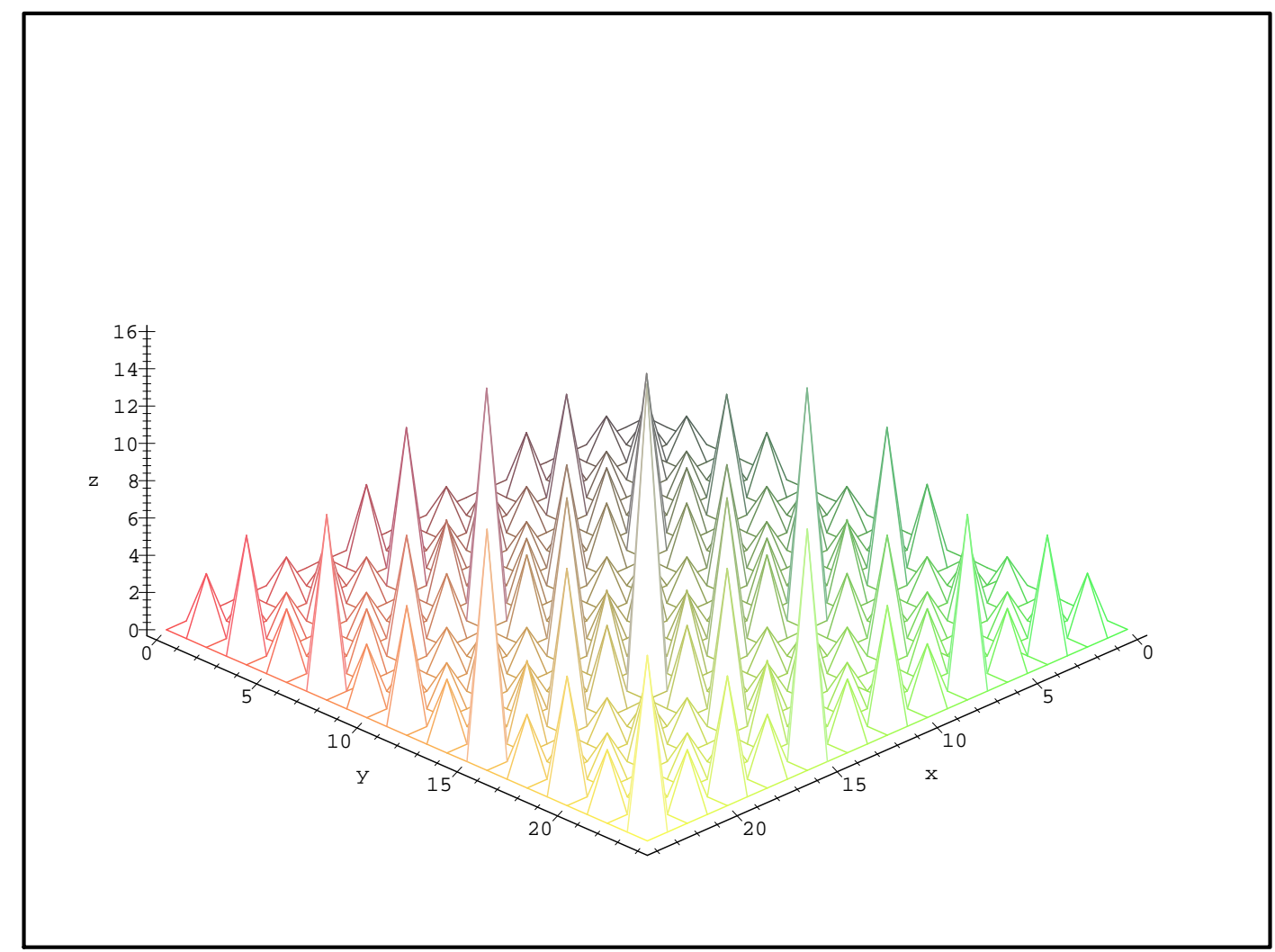

Figure 2: Matter distribution for a fixed value of the $z$-component, displaying the observed inhomogeneity.

\section{References}

[1] P.J.E. Peebles, Principles of Physical Cosmology Princeton Univ. Pr. (1993) The Large-Scale Structure of the Universe, Princeton University Press, 1980; Marc Davis, Is the Universe Homogeneous at Large Scales? Princeton 1996, Critical dialogues in cosmology* 13-23, astro-ph/9610149. 


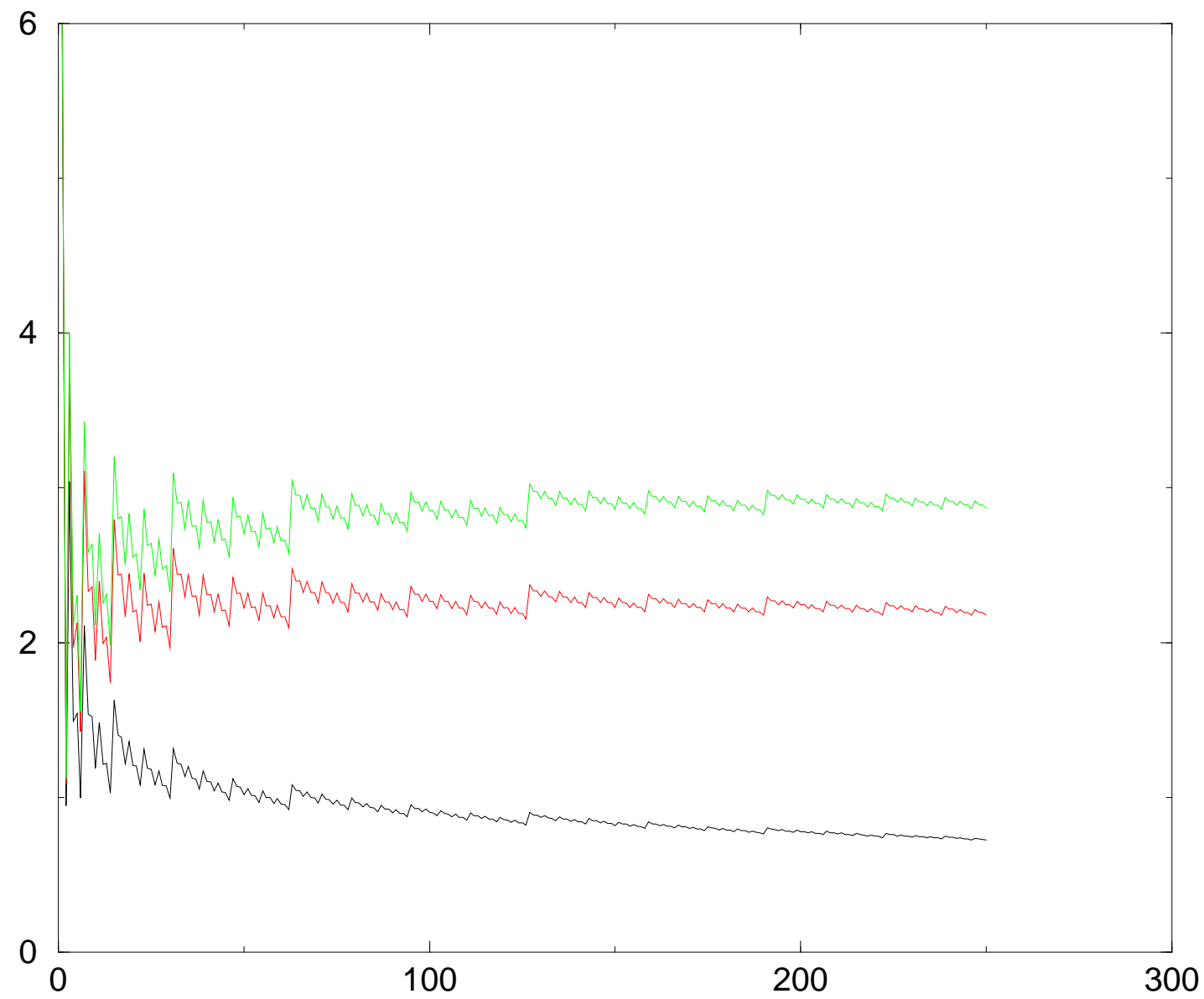

Figure 3: Evolution of the average density as further data are included, according to the limit procedure reflimit. The lower diagram corresponds to $d=3.25$, the next one to $d=3.05$, and the highest one to $d=3.00$. We note that the highest one corresponds to a constant, thus giving the correct value of $d$. We also note the fact that we need a long time to achieve a cpnstant value for the limit.

[2] P. H. Coleman and L. Pietronero Phys. Rep. 231 (1992) 311.

[3] G. Lemaitre Ann. Soc. Sci. Bruxelles A53 (1933) 51; R. C. Tolman 
Proc. Nat. Acad. Sci. USA 20 (1934) 169; H. Bondi Mon. Not. Roy. Astr. Soc. 107 (1947) 410.

[4] A. Krasiński Inhomogeneous Cosmological Models, Cambridge University Press 1997.

[5] M. B. Ribeiro Astrophysical Journal 388 (1992) 1, 395 (1992) 29, 415 (1993) 469.

[6] E. Abdalla, N. Afshordi, K. Khodjasteh and R. Mohayaee Astr. and Astro. 345 (1999) 22, astro-ph/9803187.

[7] Elcio Abdalla and M. Reza Rahimi Tabar Phys. Lett. B440 (1998) 339344, hep-th/9803161.

[8] E. Abdalla and R. Mohayaee Phys. Rev. D59 (1999) 084014, astro-ph/ 9810146 Braz. J. Phys. 31 (2001) 42-44, astro-ph/9811119.

[9] M. B. Ribeiro, Gen. Rel. Grav. 33 (2001) 1699, astro-ph/0104181.

[10] E. Abdalla, M. B. Ribeiro and R. Mohayaee Fractals 9 (2001) 451-462, astro-ph/9910003

[11] S. Weinberg Gravitation and Cosmology, John Wiley and Sons, 1972.

[12] B. B. Mandelbrot, The Fractal Geometry of Nature, Freeman, New York, 1983 